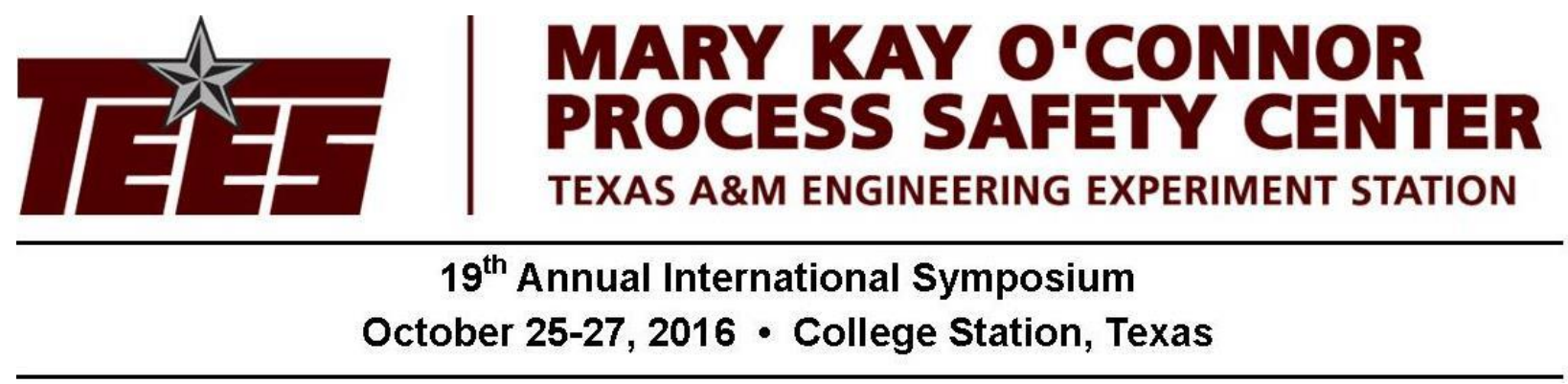

\title{
A Global Stochastic Programming Approach for the Optimal Placement of Gas Detectors with Nonuniform Unavailabilities
}

\author{
Jianfeng Liu ${ }^{1}$, Carl D. Laird ${ }^{2}$
}

\begin{abstract}
Optimal design of a gas detection systems is challenging because of the numerous sources of uncertainty, including weather and environmental conditions, leak location and characteristics, and process conditions. Rigorous CFD simulations of dispersion scenarios combined with stochastic programming techniques have been successfully applied to the problem of optimal gas detector placement; however, rigorous treatment of sensor failure and nonuniform unavailability has received less attention. To improve reliability of the design, this paper proposes a problem formulation that explicitly considers nonuniform unavailabilities and all backup detection levels. The resulting sensor placement problem is a large-scale mixed-integer nonlinear programming (MINLP) problem that requires a tailored solution approach for efficient solution. We have developed a multitree method which depends on iteratively solving a sequence of upper-bounding master problems and lower-bounding subproblems. The tailored global solution strategy is tested on a real data problem and the encouraging numerical results indicate that our solution framework is promising in solving sensor placement problems.
\end{abstract}

\section{Introduction}

To rapidly detect release events and minimize the corresponding damages, efficient detection and mitigation depends on appropriate design of the gas detector system, including type, number, and placement of sensors. However, the optimal design of these systems is very challenging because significant uncertainty must be taken into account, including weather and environmental conditions, leak location and characteristics, and process conditions. Prescriptive or semiquantitative approaches have been widely used in detector system design [15,17,22]; however, these heuristic techniques do not make full use of the information from dispersion simulations and fail to provide a rigorous proof of the solution quality.

${ }^{1}$ Ph.D. Candidate, School of Chemical Engineering, Purdue University

2 Assoc. Professor, School of Chemical Engineering, Purdue University; PMTS, Discrete Mathematics and Optimization, Sandia National Laboratories 
A number of stochastic programming (SP) approaches have been proposed and implemented to solve various real-world sensor placement problems $[1,5,6,19,20]$. Most of these previously proposed SP formulations have assumed perfect sensors. In reality, however, detectors are imperfect and subject to unpredictable failures caused by, for example, poor maintenance, erroneous calibration, or power outage. In many practical cases, these imperfections can significantly impact the performance of the entire detector system. To improve the reliability of the optimal design, therefore, it is important and necessary to explicitly consider sensor imperfection, which is measured in terms of unavailability, i.e., the probability of a false-negative detection. Berry et al. [5] first proposed an SP-based imperfect-sensor model for the contamination warning system design in water networks. Inspired by this research, Benavides-Serrano et al. [1,3] presented SP formulations for flammable gas detection and mitigation systems considering sensor unavailabilities. However, the resulting sensor placement problems, formulated as large-scale mixed-integer nonlinear programming (MINLP) problems, are very challenging to solve due to the presence of nonlinearities and discrete variables. To solve these problems efficiently, Benavides-Serrano et al. [3] approximated the MINLP as a mixed-integer quadratic programming problem (MIQP) by considering only one or two levels of redundancy. In this paper, we provide a rigorous problem formulation and solution approach for optimal sensor placement that does not require this approximation.

Sensor placement problems considering nonuniform unavailabilities are formulated as MINLP problems. Two major categories of solution techniques have been used to solve MINLP problems. Stochastic approaches, such as random search, simulated annealing, and genetic algorithms, can be easily implemented, however, none of these algorithms can provide a guarantee of the solution optimality. Deterministic methods, in contrast, are able to provide a rigorous mathematical guarantee of global optimality. The single-tree deterministic algorithms, such as the well-known branch-and-bound (BB) methods [7,18], have been well-studied and intensively extended to a variety of global optimization algorithms, such as Branch-and-Reduce [25], Reduced Space Branch-and-Bound [9], Branch-and-Contract [30], Branch-and-Cut [14] and Branch-andSandwich [16]. In general, these techniques are suitable for non-convex MINLP problems of small or medium size. Alternatively, multitree methods are based on iteratively solving a sequence of master problems and slave problems (or subproblems) $[8,29]$. To handle 'non-convex' MINLP problems with certain special features, e.g., bilinear, posynomial, linear fractional, and concave separable, extensions of these well-studied multitree methods can be found in literature [23,24].

The proposed global optimization algorithm is regarded as a multitree method, since it is an iterative algorithm relying on solutions of a sequence of mixed-integer master problems and nonlinear subproblems. The master problem, which formulated as a mixed-integer linear programming (MILP) problem, is a strict convex relaxation of the MINLP problem formulation. To obtain a relatively tight and computationally efficient master problem, we introduce linear outer approximations and tight, problem specific, upper bounding constraints. The upper bounding subproblem is obtained by fixing all binary variables, which, in this case, results in a subproblem that can be directly computed with a single forward simulation. In this way, iterations cycle between the solution of the master problem and the subproblem, generating a sequence of lower and upper bounds. The global algorithm terminates when the relative optimality gap is below a given tolerance. 
The rest of this paper is organized as follows. In Section 2, we briefly review mathematical models for sensor placement problems. Section 3 presents our new problem formulation based on the logtransformation of the original problem formulation from [5]. Section 4 outlines our tailored global solution framework and master problems formulated as mixed-integer linear programming problems. Sections 5 shows numerical results on a real data test problem and Section 6 provides summary and conclusions.

\section{Background}

In this section, we provide a brief review of the literature on optimal sensor placement. The sensor placement problem can be regarded as a special case of the p-median problem (PMP). In a PMP, we want to locate $p$ facilities to minimize the weighted average distance between the demand nodes and the nearest of the selected facilities [12]. The optimal sensor placement problem assuming perfect sensors is equivalent to a classic PMP, and well-known solution strategies designed for PMPs can be directly implemented, including heuristic and greedy algorithms. However, most of these strategies may fail to guarantee high solution quality. The stochastic programming (SP) approach, on the other hand, provides an alternative way to solve for a PMP. Particularly, the first SP formulation for sensor placement problem in water network systems was proposed by Berry et al. [6]. With the assumption of perfect sensors, the resulting optimization problem is formulated as an MILP problem, which can be solved by general mixed-integer solvers.

Detectors are, however, imperfect and subject to unpredictable failures. In many cases, these imperfections can significantly impact the performance of the entire detector system. To improve the reliability of the entire detection system, therefore, it is necessary to explicitly consider sensor unavailability, i.e., the probability of a false-negative detection. A number of extensions to the original PMPs have been proposed to handle facility unavailabilities. For instance, Snyder and Daskin [26] presented the reliability PMP (RPMP) based on the assumption of uniform unavailabilites. In this approach, the probability products are modeled via the binomial distribution, which leads to an MILP problem. The median problem with unreliable facilities (MPUF) is proposed by Berman et al. [4], where the unvailabilities are assumed to be uniform and the detection levels are limited to a given number. For a comprehensive review of the unavailability considerations into the PMPs please refer to Benavides-Serrano et al [1] and Snyder et al. [27].

In the context of stochastic programming approaches, the first imperfect-sensor model is presented by Berry et al. [5]. This model, though originally proposed to design the contaminant warning systems in water networks, is general and well-suited for any sensor placement problem considering unavailabilities. However, the resulting MINLP problem is very difficult to solve due to strong nonlinearities. To partially address this challenge, previous work has assumed uniform unavailability across all sensors in the network [1]. However, this assumption is not always reasonable since the probability of sensor failure usually depends on the detector type, maintenance condition, and environment. An alternative approach was recently proposed by Benavides-Serrano et al. [3] based on reducing the number of detection levels while maintaining nonuniform unavailabilities. Sensitivity analysis has shown that for small-to-moderate unavailabilities we only need to consider a small number of detection levels. While effective under 
these assumptions, it is worthwhile to point out that these SP formulations are approximations of the original sensor placement problem. As a result, none of these approaches can provide a rigorous guarantee of solution quality to the original MINLP problem. Moreover, to the best of our knowledge, there exists no solution framework that is designed to solve a general sensor placement problem with nonuniform unavailabilites to global optimality. In this paper, we extend previous problem formulations and present a multitree solution strategy based on tailored relaxations of the MINLP problem.

\section{Stochastic Programming Formulations and Solution Approach}

The imperfect-sensor SP model, labeled impSP, was originally proposed by Berry et al. [5] to design contaminate warning systems for water networks.

$$
\begin{aligned}
& \min \sum_{a \in A} \alpha_{a} \sum_{i \in L_{a}} d_{a, i} x_{a, i} \\
& \text { s.t. } \sum_{i \in L_{a}} x_{a, i}=1 \quad \forall a \in A \\
& x_{a, i}=\left(1-\bar{q}_{i}\right) s_{i} \prod_{j \in L_{a, i}}\left(1-\left(1-\bar{q}_{j}\right) s_{j}\right) \quad \forall a \in A, i \in L_{a} \backslash\{D\} \\
& \sum_{i \in L \backslash\{D\}} s_{i} \leq p \\
& 0 \leq x_{a, i} \leq 1 \quad \forall a \in A, i \in L_{a} \\
& s_{i} \in\{0,1\} \quad \forall i \in L
\end{aligned}
$$

The complete list of symbols for this problem formulation are described in Table 1. Here, $A$ presents the set of hazardous scenarios, and $L$ presents the set of all candidate detector locations. The goal is to select a sensor placement that minimizes the expected value of the damage across all the scenarios. The parameter $\alpha_{a}$ is the probability (or weight) of scenario $a$, which is obtained from the scenario distribution based on the historical data or computer-aided simulations. Parameter $d_{a, i}$ is the damage coefficient, which, for these studies, is the detection time of scenario $a$ at location $i$. Typically, and in this study, these damage coefficients are estimated from computational fluid dynamics (CFD) simulations. Further description of this problem formulation can be found in Benavides-Serrano et al [3] and Berry et al. [5].

\section{Table 1: Notation}

\begin{tabular}{|c|l|}
\hline Sets & \\
\hline $\boldsymbol{A}$ & Set of hazardous scenarios \\
\hline $\boldsymbol{L}$ & Set of candidate detector locations \\
\hline $\boldsymbol{L}_{\boldsymbol{a}}$ & Set of locations that can detect scenario $a$ \\
\hline $\boldsymbol{L}_{\boldsymbol{a}, \boldsymbol{i}}$ & Set of locations that witness scenario $a$ better (in terms of damage) than $i$ \\
\hline Parameters & \\
\hline $\boldsymbol{\alpha}_{\boldsymbol{a}}$ & Probability of scenario $a$ \\
\hline
\end{tabular}




\begin{tabular}{c|l}
\hline $\boldsymbol{d}_{\boldsymbol{a}, \boldsymbol{i}}$ & Damage coefficient for scenario $a$ if detected by location $i$ \\
$\overline{\boldsymbol{q}}_{\boldsymbol{i}}$ & Time-averaged unavailability for detector placed at location $i$ \\
\hline $\boldsymbol{p}$ & Maximum number of detectors allowed \\
\hline Variables & \\
\hline $\boldsymbol{x}_{\boldsymbol{a}, \boldsymbol{i}}$ & Probability that a detector at location $i$ is the first to detect scenario $a$ \\
\hline$\tilde{\boldsymbol{x}}_{\boldsymbol{a}, \boldsymbol{i}}, \overline{\boldsymbol{x}}_{\boldsymbol{a}, \boldsymbol{i}}$ & Intermediate variables in our alternate formulation \\
\hline $\boldsymbol{s}_{\boldsymbol{i}}$ & Binary variable equal to 1 if a detector is placed at location $i$, and zero otherwise \\
\hline $\boldsymbol{z}_{\boldsymbol{i}, \boldsymbol{j}}$ & Aggregated variable equal to $s_{i} s_{j}$ for $i \neq j$ \\
\hline
\end{tabular}

The continuous variable $x_{a, i}$ is the probability that the detector placed at location $i$ will be the first to detect hazardous scenario $a$. The binary variable $s_{i}$ is an indicator for a detector placed at location $i$. If a detector is installed at location $i$ then $s_{i}=1$, and otherwise $s_{i}=0$. Subset $L_{a} \subseteq L$ is the set of candidate locations that can provide detection of hazardous scenario $a$. For a particular scenario, however, it is possible that no candidate location can provide detection, therefore, a dummy location $D$ is also included with a sufficiently large damage coefficient, to account for the impact of an undetected scenario. Subset $L_{a, i}$ is the set of candidate locations that can witness scenario $a$ better (in terms of detection time or another damage metric) than location $i$.

The first constraint in (impSP) guarantees that the summation of the probabilities equals 1 for each scenario (recall that a dummy detector is included). For each non-dummy location, the second constraint provides the expression for the probability $x_{a, i}$, where $\bar{q}_{i}$ is the given time-averaged unavailability of a detector placed at location $i$. This probability constraint is strongly nonlinear due to the product of the binary variables. Note that, due to the first constraint, the probability of detection by the dummy location, $x_{a, D}$, also provides the probability that all detectors fail to report a hazardous scenario. The third constraint provides an upper bound of the total number of detectors that can be allocated in the system (not including the dummy detector).

\subsection{Alternative MINLP Formulation}

The original formulation (impSP) is strongly nonlinear due to the multiplication of binary variables in the relationship for probability $x_{a, i}$. We propose an alternative formulation, which is mathematically equivalent to the original formulation, based on the log-transformation of the probability equation.

First, we define a new binary variable $z_{i, j}$ as,

$$
z_{i, j} \equiv s_{i} s_{j} \quad \forall i, j \in L, i \neq j
$$

Note that $z_{i, j}$ indicates if gas detectors are placed at both location $i$ and location $j$. That is $z_{i, j}=1$ if and only if both $s_{i}=1$ and $s_{j}=1$. This logic relationship can be expressed in terms of a set of linear inequalities

$$
\begin{array}{ll}
s_{i}+s_{j}-1 \leq z_{i, j} & \forall i, j \in L, i \neq j \\
z_{i, j} \leq s_{i} & \forall i, j \in L, i \neq j \\
z_{i, j} \leq s_{j} & \forall i, j \in L, i \neq j
\end{array}
$$


Fortunately, given the fact that $s_{i}$ is a binary variable, imposing these constraints guarantees that $z_{i, j}$ solves to a binary value, and it can be relaxed as a continuous variable within the range of 0 to 1 .

The log-transformation of the nonlinear probability constraint can be rewritten as a linear equality constraint

$$
\tilde{x}_{a, i}=s_{i} \ln \left(1-\bar{q}_{i}\right)+\sum_{j \in L_{a, i}} z_{i, j} \ln \left(\bar{q}_{j}\right) \quad \forall a \in A, i \in L_{a} \backslash\{D\}
$$

However, the new variable $\tilde{x}_{a, i}$ is not an exact $\log$-transformation of the probability $x_{a, i}$. For instance, if $s_{i}=0$, meaning that no detector is installed at location $i$, we have both $x_{a, i}=0$ from formulation (impSP) and $\tilde{x}_{a, i}=0$ from equations (1) and (2), while the exact log-transformation of $x_{a, i}$ gives $-\propto$. To recover $x_{a, i}$ from $\tilde{x}_{a, i}$, we need to introduce a new variable $\bar{x}_{a, i}$ and two additional constraints

$$
\begin{array}{ll}
\bar{x}_{a, i}=\exp \left(\tilde{x}_{a, i}\right) & \forall a \in A, i \in L_{a} \\
x_{a, i}=\bar{x}_{a, i}+s_{i}-1 & \forall a \in A, i \in L_{a}
\end{array}
$$

A mathematically equivalent formulation of the original formulation (impSP) is then given as

$$
\begin{aligned}
& \min \sum_{a \in A} \alpha_{a} \sum_{i \in L_{a}} d_{a, i} x_{a, i} \\
& \text { s.t. } \sum_{i \in L_{a}} x_{a, i}=1 \quad \forall a \in A \\
& \tilde{x}_{a, i}=s_{i} \ln \left(1-\bar{q}_{i}\right)+\sum_{j \in L_{a, i}} z_{i, j} \ln \left(\bar{q}_{j}\right) \quad \forall a \in A, i \in L_{a} \backslash\{D\} \\
& \bar{x}_{a, i}=\exp \left(\tilde{x}_{a, i}\right) \quad \forall a \in A, i \in L_{a} \\
& x_{a, i}=\bar{x}_{a, i}+s_{i}-1 \quad \forall a \in A, i \in L_{a} \\
& s_{i}+s_{j}-1 \leq z_{i, j} \quad \forall i, j \in L, i \neq j \\
& z_{i, j} \leq s_{i} \quad \forall i, j \in L, i \neq j \\
& z_{i, j} \leq s_{j} \quad \forall i, j \in L, i \neq j \\
& \sum_{i \in L \backslash\{D\}} s_{i} \leq p \\
& 0 \leq x_{a, i} \leq 1 \quad \forall a \in A, i \in L_{a} \\
& s_{i} \in\{0,1\} \quad \forall i \in L \\
& 0 \leq z_{i, j} \leq 1 \quad \forall i, j \in L, i \neq j
\end{aligned}
$$

We label this (impSP-LT) to indicate that it is an exact log-transformation of the original formulation (impSP). This alternative formulation, nevertheless, is still an MINLP problem due to binary variables and exponential terms. Compared with the original formulation, however, the reformulation is mathematically preferable since its nonlinearity only arises from convex 
univariate functions. This property facilitates straightforward development of our global solution strategy.

\subsection{MINLP Solution Algorithm}

Regarded as a multitree method, our global solution framework is an iterative algorithm relying on solving a sequence of lower bounding master problems and upper bounding subproblems. The master problem, formulated as an MILP problem, is a relaxation of the sensor placement problem formulated in (impSP-LT). If the master problem is infeasible, the corresponding sensor placement problem is also infeasible and the algorithm terminates. Otherwise, the master problem provides a valid lower bound and a candidate set of values for the discrete decisions (the sensor placement, $s_{i}$ ). The mixed-integer master problem can be refined by several techniques discussed later. A corresponding upper-bounding subproblem is obtained by fixing all binary variables present in the formulation to the values from the solution of the master problem. In this particular formulation, when all binary variables are specified, the resulting upper-bounding subproblem is square and can be computed directly. This subproblem provides a valid upper bound of the sensor placement problem. The algorithm proceeds through a series of major iterations, cycling between the solution of a mixed-integer master problem (for the lower bound) and a forward simulation of the upperbounding subproblem, yielding a sequence of lower and upper bounds. Finally, the algorithm terminates when the relative optimality gap is below a given tolerance.

This solution approach requires an effective relaxation of (impSP-LT) that can be refined to produce tighter and tigher lower bounds. We present a strict convex relaxation of the formulation by introducing linear outer approximations. This relaxation, formulated as an MILP problem, is used as the lower bounding master problem in our global optimization algorithm. Notice in the alternative formulation (impSP-LT), the nonlinearity only arises from the univariate convex function $\exp \left(\tilde{x}_{a, i}\right)$. While these exponential terms can be relaxed using linear under- and piecewise linear over-estimators, this approach would introduce additional binary variables (or SOS2 constraints) for the piecewise linear over-estimators. Furthermore, the number of binary variables increases as we increase the number of segments during refinement.

\subsubsection{MILP Relaxation with Tight Upper Bounding Constraints}

To avoid using the piecewise linear over-estimators, we propose instead a tailored approach and introduce a different convex relaxation without these piecewise linear functions. Given the objective function and positive damage coefficients $d_{a, i}$ the optimization problem tends to push all $x_{a, i}$ to the lowest possible value so that the object function value is minimized. However, the first constraint in formulation (impSP-LT) requires that the probabilities for each scenario sum to 1 , therefore locations with larger damage coefficients will still be pushed down to their lower bounds, while the locations with small damage coefficients will be pushed to their upper bound. Therefore, the upper bounds of $x_{a, i}$, especially those of the locations with relatively low damage coefficients can have a strong impact on the tightness of the convex relaxation.

Here, we use the concept of detection levels proposed by Benavides-Serrano et al [3]. In particular, if a sensor is at detection level $N$ for a scenario, then for this detector to be the first to detect the scenario, all $N-1$ detectors at better locations (with smaller damage coefficients) must of failed to operate correctly. For instance, if a detector is placed at a location with the smallest damage 
coefficients compared with the others, then it is at the first detection level, and the value of $x_{a, i}=$ $1-\bar{q}_{i}$. For the second detection level, the probability is given by $x_{a, i}=\bar{q}_{j}\left(1-\bar{q}_{i}\right)$, where $j$ and $i$ are the locations with the smallest and the second smallest damage coefficients for scenario $a$, respectively. With this knowledge, we can write the upper bound of $x_{a, i}$ up to two detection levels as,

$$
\bar{x}_{a, i} \leq\left(1-\bar{q}_{i}\right)\left(s_{i}+\left(\bar{q}_{j}-1\right) z_{i, j}\right) \quad \forall a \in A, i \in L_{a}, j \in L_{a, i}
$$

Note if there is no detector installed at location $i$, i.e., $s_{i}=0$, then $x_{a, i}$ is forced to be 0 . If $s_{i}=1$ and location $i$ happens to be at the first detection level for scenario $a$ then we have $x_{a, i} \leq 1-\bar{q}_{i}$. Recall the fact that at the optimal solution for $x_{a, i}$ corresponding to the smallest damage coefficient will be lifted up to its upper bound and we have that $x_{a, i}=1-\bar{q}_{i}$. In other words, this upper bound is active and thus the tightest. Similar arguments hold true for higher detection levels, and the upper bounding constraints shown above are the tightest for the first two detection levels and provide valid upper bounds at higher detection levels.

We impose the upper bounding constraints leading to the relaxation,

$$
\begin{array}{lll}
\min & \sum_{a \in A} \alpha_{a} \sum_{i \in L_{a}} d_{a, i} x_{a, i} & \\
\text { s.t. } & \sum_{i \in L_{a}} x_{a, i}=1 & \\
& \forall a \in A \\
\tilde{x}_{a, i}=s_{i} \ln \left(1-\bar{q}_{i}\right)+\sum_{j \in L_{a, i}} z_{i, j} \ln \left(\bar{q}_{j}\right) & \forall a \in A, i \in L_{a} \backslash\{D\} \\
& \\
x_{a, i} \leq\left(1-\bar{q}_{i}\right)\left(s_{i}+\left(\bar{q}_{j}-1\right) z_{i, j}\right) & \forall a \in A, i \in L_{a}, j \in L_{a, i} \\
\bar{x}_{a, i} \geq \overline{\exp }\left(\tilde{x}_{a, i, m}^{*}\right)\left(\tilde{x}_{a, i}-\tilde{x}_{a, i, m}^{*}+1\right) & \forall a \in A, i \in L_{a}, m \in M_{a, i} \\
x_{a, i}=\bar{x}_{a, i}+s_{i}-1 & \forall a \in A, i \in L_{a} \\
s_{i}+s_{j}-1 \leq z_{i, j} & \forall i, j \in L, i \neq j \\
z_{i, j} \leq s_{i} & \forall i, j \in L, i \neq j \\
z_{i, j} \leq s_{j} & \forall i, j \in L, i \neq j \\
\sum_{i \in L \backslash\{D\}} s_{i} \leq p & \\
0 \leq x_{a, i} \leq 1 & \forall a \in A, i \in L_{a} \\
s_{i} \in\{0,1\} & \forall i \in L \\
0 \leq z_{i, j} \leq 1 & \forall i, j \in L, i \neq j
\end{array}
$$

Here $\overline{\exp }$ refers to linearizations of the function, forming valid under-estimators. There are two ways to further improve the tightness of the relaxation (impSP-R). First, we can increase the number of segment points to refine the linear under-estimators. Second, we can impose similar 
upper bounding constraints for higher detection levels. For instance, tight upper bounding constraints up to three detection levels can be written as,

$x_{a, i} \leq\left(1-\bar{q}_{i}\right)\left(s_{i}+\left(\bar{q}_{j}-1\right) z_{i, j}+\bar{q}_{j}\left(\bar{q}_{k}-1\right)\left(z_{i, j}+z_{j, k}-1\right)\right) \quad \forall a \in A, i \in L_{a}, j \in L_{a, i}, k \in L_{a, j}$.

Note that no additional binary variables are required in generating tighter upper bounding constraints. Theoretically, we can impose these upper bounding constraints to arbitrary detection levels. However, the number of these constraints may increase dramatically and the resulting relaxation problem can soon become prohibitively large. Moreover, the tightness may not be significantly improved since the probability at higher detection level may be negligible.

\section{Numerical Results}

In this section, we test our MINLP formulation and global solution approach on a gas detector placement problem with nonuniform unavailabilities. First, we provide a brief introduction on the simulation data used in this paper. Then we present the computational performance of our tailored algorithm in solving the gas detector placement problem.

\subsection{Data Set}

The data set used here is previously employed by Benavides-Serrano et al. $[1,3]$ and Legg et al. $[19,20]$. It is based on a real, medium-scale, proprietary offshore facility geometry capturing the full process features, such as equipment, piping, and support structures. Gas dispersion scenario simulations were provided by GexCon, computed with FLACS, a validated tool for gas dispersion and explosion modeling in the technical safety context. Particularly, this data set consists of 270 hazard scenarios and 994 potential gas detector locations. The damage coefficient is the time between the initiation of a hazardous scenario $a$ and its detection by a gas sensor placed at location $i$. To capture undetected scenarios, the damage coefficient of the dummy location is set to a value larger than the other damage coefficients. For a complete discussion regarding the data set, the data generation, and the data collection procedure please refer to previous work [2].

\subsection{Sensor Placement Results}

The convex relaxation (impSP-R2) is used as the master problem in our global solution framework. The resulting lower bounding master problem is formulated as a mixed-integer linear programming (MILP) problem. Our tailor global solution framework is implemented in Pyomo, a Python-based optimization modeling language [13]. The MILP master problem is solved to an optimality gap below $0.001 \%$. The relative optimality gap of the global algorithm is set to be $0.01 \%$. The total computational time limit is 36,000 seconds and the outer iteration number limit is 30. The mixed-integer master problems are solved with Gurobi [21] and the forward simulations are directly computed. The computational performance of the optimization approach is shown in Table 2 for different values of the maximum number of sensors $p$. 
Table 2: Computational Performance Results

\begin{tabular}{|c|c|c|c|c|c|}
\hline$p$ & Best Solution & $\begin{array}{l}\text { Lower } \\
\text { Bound }\end{array}$ & $\operatorname{Gap}(\%)$ & CPU Time & Iteration \\
\hline 1 & 8622.88 & 8622.88 & 0 & 69 & 1 \\
\hline 5 & 5954.81 & 5981.49 & 0 & 1003 & 2 \\
\hline 10 & 4354.6 & 4357.52 & 0 & 2306 & 2 \\
\hline 15 & 3302.06 & 3303.6 & 0 & 2484 & 2 \\
\hline 20 & 2553.53 & 2554.22 & 0 & 1911 & 2 \\
\hline 25 & 1894.25 & 1894.76 & 0 & 4128 & 3 \\
\hline 30 & 1426.55 & 1426.57 & 0 & 4213 & 3 \\
\hline 35 & 1068.67 & 1068.12 & 0.0005 & 9032 & 7 \\
\hline 40 & 779.09 & 778.43 & 0.0008 & 7929 & 4 \\
\hline 45 & 581.14 & 579.87 & 0.0022 & $46636^{*}$ & 2 \\
\hline
\end{tabular}

As the number of detector increases (from 1 to 45), the object function value improves (from 8623 to 581) since more scenarios can be detected faster. As observed in other work, increasing the number of gas detectors has more impact when the number of sensors are relatively small, since more of the scenarios are detected, and the algorithm is focusing on achieving maximum coverage (to avoid the penalty of the dummy location). However, as the number of sensors increases, full coverage is achieved, and the focus is shifted to reducing the expected time to detection alone with additional detectors. Therefore, trade-offs must be made between the number of detectors and the expected detection time across all scenarios.

From a mathematical point of view, our proposed global solution framework is able to solve this sensor placement problem to global optimality. Particularly, when the number of detectors is relatively small (1 to 30), the resulting sensor placement problems are globally solved in under 2 hours. Even for larger problems, however, the solutions times are still many orders of magnitude less than the time required to compute the dispersion scenarios.

\section{Summary and Conclusions}

Gas detection and mitigation systems play very important roles in modern process safety since they can protect lives and reduce the potential damage caused by combustible and toxic gas leaks. However, the optimal design of a gas detection system is very challenging because of the inherent uncertainty such as gas compositions, leak locations, process conditions, and weather. To address this uncertainty, prescriptive or semi-quantitative approaches have been widely used for decades, however, none of these techniques can provide rigorous proof of the solution quality. To deal with this issue, there is a need to develop rigorous quantitative strategies for gas detector system design. Stochastic programming (SP) provides an appropriate mechanism for solution of these sensor placement problems.

The SP formulations proposed in this paper consider nonuniform failure probabilities. Whereas previous work considered only two backup levels (valid when unavailability values are low), arriving at a mixed-integer quadratic programming (MIQP) problem, in our formulation, all backup detection levels are explicitly taken into account. The resulting sensor placement problem 
is formulated as a large-scale mixed-integer nonlinear programming (MINLP) problem. To solve this challenging MINLP problem to global optimality, we propose a multitree method which depends on iteratively solving a sequence of lower-bounding master problems and upper-bounding subproblems. The upper-bounding subproblems can be directly computed (they become square once the binaries are fixed). The master problem, however, is a mixed-integer problem that must be formulated as a convex relaxation of the original MINLP problem. To obtain a relatively tight and computationally efficient master problem, we first propose an equivalent log-transformation of the original MINLP formulation. Though new variables and constraints are introduced, this alternative formulation is mathematically preferable since all its nonlinearity comes from convex univariate terms. Based on this reformulation, we present a strictly convex relaxation by introducing linear outer approximations and tight upper bounding constraints. The resulting relaxation formulated as a mixed-integer linear programming (MILP) problem is used as the lowerbounding master problem. The proposed global solution strategy is tested on a number of real data problems and the encouraging numerical results indicate that this solution framework is computationally feasible for large datasets.

\section{Acknowledgments}

The authors would like to thank Purdue Process Safety and Assurance Center (P2SAC) for their continued support of this research. The authors would also like to thank GexCon for their support in providing dispersion simulation data for this paper. Sandia National Laboratories is a multiprogram laboratory managed and operated by Sandia Corporation, a wholly owned subsidiary of Lockheed Martin Corporation, for the U.S. Department of Energy's National Nuclear Security Administration under contract DE-AC04-94AL85000.

\section{References}

[1] A.J. Benavides-Serrano, S.W. Legg, R Vazquez-Roman, M.S. Mannan, and C.D. Laird. A stochastic programming approach for the optimal placement of gas detectors: Unavailability and voting strategies. Industrial \& Engineering Chemistry Research, 53(13):5355-5365, 2013. [2] A.J. Benavides-Serrano, M.S. Mannan, and C.D. Laird. A quantitative assessment on the placement practices of gas detectors in the process industries. Journal of Loss Prevention in the Process Industries, 35:339-351, 2015.

[3] A.J. Benavides-Serrano, M.S. Mannan, and C.D. Laird. Optimal placement of gas detectors: A p-median formulation considering dynamic nonuniform unavailabilities. AIChE Journal, 2016.

[4] Oded Berman, Dmitry Krass, and Mozart BC Menezes. Facility reliability issues in network p-median problems: Strategic centralization and co-location effects. Operations Research, 55(2):332-350, 2007.

[5] J. Berry, R.D. Carr, W.E. Hart, V.J. Leung, C.A. Phillips, and J. Watson. Designing contamination warning systems for municipal water networks using imperfect sensors. Journal of Water Resources Planning and Management, 135(4):253-263, 2009.

[6] J.W. Berry, L. Fleischer, W.E. Hart, C.A. Phillips, and J. Watson. Sensor placement in municipal water networks. Journal of Water Resources Planning and Management, 131(3): 237-243, 2005.

[7] Robert J. Dakin. A Tree-Search Algorithm for Mixed Integer Programming Problems. The Computer Journal, 8(3):250-255, 1965. 
[8] Marco A. Duran and Ignacio E. Grossmann. An Outer-Approximation Algorithm for a Class of Mixed-Integer Nonlinear Programs. Mathematical programming, 36(3):307-339, 1986.

[9] Thomas GW Epperly and Efstratios N. Pistikopoulos. A reduced Space Branch and Bound Algorithm for Global Optimization. Journal of Global Optimization, 11(3):287-311, 1997. [10] Bjorn Geißler, Alexander Martin, Antonio Morsi, and Lars Schewe. Using piecewise linear functions for solving minlps. In Mixed Integer Nonlinear Programming, pages 287-314. Springer, 2012.

[11] Arthur M. Geoffrion. Generalized Benders Decomposition. Journal of optimization theory and applications, 10(4):237-260, 1972.

[12] S Louis Hakimi. Optimum distribution of switching centers in a communication network and some related graph theoretic problems. Operations Research, 13(3):462-475, 1965. [13] William E Hart, Carl Laird, Jean-Paul Watson, and David L. Woodruff. PyomoOptimization Modeling in Python, volume 67. Springer Science \& Business Media, 2012 [14] Padmanaban Kesavan and Paul I. Barton. Generalized Branch-and-Cut Framework for Mixed-Integer Nonlinear Optimization Problems. Computers \& Chemical Engineering, 24 (2):1361-1366, 2000.

[15] Avner Kessler, Avi Ostfeld, and Gideon Sinai. Detecting accidental contaminations in municipal water networks. Journal of Water Resources Planning and Management, 124(4): 192-198, 1998.

[16] Polyxeni-Margarita Kleniati and Claire S. Adjiman. Branch-and-Sandwich: A Deterministic Global Optimization Algorithm for Optimistic Bilevel Programming Problems. Part I: Theoretical Development. Journal of Global Optimization, pages 1-34, 2014.

[17] Arun Kumar, ML Kansal, Geeta Arora, Avi Ostfeld, and Avner Kessler. Detecting accidental contaminations in municipal water networks. Journal of Water Resources Planning and Management, 125(5):308-310, 1999.

[18] Ailsa H. Land and Alison G. Doig. An Automatic Method of Solving Discrete Programming Problems. Econometrica: Journal of the Econometric Society, pages 497-520, 1960.

[19] S.W. Legg, A.J. Benavides-Serrano, J.D. Siirola, J. Watson, S.G. Davis, A. Bratteteig, and C.D. Laird. A stochastic programming approach for gas detector placement using cfdbased dispersion simulations. Computers \& Chemical Engineering, 47:194-201, 2012. [20] S.W. Legg, C. Wang, A.J. Benavides-Serrano, and C.D. Laird. Optimal gas detector placement under uncertainty considering conditional-value-at-risk. Journal of Loss Prevention in the Process Industries, 26(3):410-417, 2013.

[21] Gurobi Optimization and Others. Gurobi optimizer reference manual. URL: http://www. gurobi. com, 2012.

[22] Avi Ostfeld and Elad Salomons. Optimal layout of early warning detection stations for water distribution systems security. Journal of Water Resources Planning and Management, 130 (5):377-385, 2004.

[23] Ray Porn and Tapio Westerlund. A Cutting Plane Method for Minimizing Pseudo-Convex Functions in the Mixed Integer Case. Computers \& Chemical Engineering, 24(12):2655$2665,2000$. 
[24] Ray Porn, Iiro Harjunkoski, and Tapio Westerlund. Convexification of Different Classes of Non-Convex MINLP Problems. Computers \& chemical engineering, 23(3):439-448, 1999. [25] Hong S. Ryoo and Nikolaos V. Sahinidis. A Branch-and-Reduce Approach to Global Optimization. Journal of Global Optimization, 8(2):107-138, 1996.

[26] Lawrence V Snyder and Mark S Daskin. Reliability models for facility location: the expected failure cost case. Transportation Science, 39(3):400-416, 2005.

[27] Lawrence V Snyder, Zumbul Atan, Peng Peng, Ying Rong, Amanda J Schmitt, and Burcu Sinsoysal. Or/ms models for supply chain disruptions: A review. IIE Transactions, pages 121,2015

[28] Juan Pablo Vielma, Shabbir Ahmed, and George Nemhauser. Mixed-integer models for non- separable piecewise-linear optimization: Unifying framework and extensions. Operations research, 58(2):303-315, 2010.

[29] Tapio Westerlund and Frank Pettersson. An Extended Cutting Plane Method for Solving Convex MINLP Problems. Computers \& Chemical Engineering, 19:131-136, 1995.

[30] Juan M. Zamora and Ignacio E. Grossmann. A Branch and Contract Algorithm for Problems with Concave Univariate, Bilinear and Linear Fractional Terms. Journal of Global Optimization, 14(3):217-249, 1999. 